\title{
Signatures of Anderson localization in the ionization rates of periodically driven Rydberg states
}

\author{
Sandro Wimberger and Andreas Buchleitner \\ Max-Planck-Institut für Physik komplexer Systeme, Nöthnitzer Str. 38, D-01187 \\ Dresden \\ e-mail: saw@mpipks-dresden.mpg.de
}

\begin{abstract}
We provide a statistical characterization of the ionization yield of onedimensional, periodically driven Rydberg states of atomic hydrogen, in the spirit of Anderson localization theory. We find excellent agreement with predictions for the conductance across an Anderson localized, quasi one-dimensional, disordered wire, in the semiclassical limit of highly excited atomic initial states. For the moderate atomic excitations typically encountered in state of the art laboratory experiments, finite-size effects induce significant deviations from the solid-state picture. However, large scale fluctuations of the atomic conductance prevail and are robust when averaged over a finite interval of driving field amplitudes, as inevitably done in the experiment.

PACS numbers: $72.15 . \mathrm{Rn}, 05.45 . \mathrm{Mt}, 32.80 . \mathrm{Rm}, 42.50 . \mathrm{Hz}$
\end{abstract}

\section{Introduction}

Coherent quantum transport on a mesoscopic scale is the origin of many intriguing transport phenomena in complex systems [1]. The somewhat vague attribute "complex" summarizes a multitude of more specific physical situations: complex dynamics can be generated by disorder, by many-particle interactions, and by dynamical chaos, to name a few.

Arguably one of the most prominent and most fundamental coherence effects in complex quantum transport is Anderson localization [2], the quantum suppression of conductance across a disordered, quasi one-dimensional solid-state lattice. Viewed as a scattering problem, it essentially manifests itself in exponentially small transmission probabilities from input to output of the sample, as a consequence of multiple scattering events (with finite reflection and transmission coefficients) at randomly placed scattering sites along the lattice. This naturally generates a multitude of transmission amplitudes which have to be summed up coherently on output. If their individual phases have been randomized by the disordered lattice potential, they will tend to interfere destructively. In terms of electronic eigenfunctions, Anderson localization enforces their exponential localization on the lattice domain. The degree of localization is characterized by the localization length $\xi$, which should be compared to the sample size $L$, in order to allow 
predictions on the conductance across the sample [3]. Complexity is brought about in this problem by two components: (a) the large number of interfering transmission amplitudes, and (b) the disordered lattice potential which breaks the translational invariance (which otherwise would reduce the complexity introduced by (a) through some kind of Bragg condition).

As mentioned before, complexity can have different causes, and therefore a random potential is not necessary to enforce vanishing total transition amplitudes or exponentially localized eigenfunctions - any mechanism which equidistributes the phases of the individual interfering amplitudes will do. In particular, dynamical chaos can substitute for disorder in quantum systems with a well-defined classical counterpart, and more generally, if such an analogy is unavailable, deterministic quantum systems with largely broken symmetries or non-perturbatively coupled degrees of freedom (displaying quantum chaos) can replace the simple scenario of a disordered lattice (there is a caveat concerning the dimensionality of the dynamics, but we shall restrain here to effectively one-dimensional systems) [4, 5, 6, 7]. The only further ingredient required for such systems to mimic Anderson's scenario is a sufficiently high density of states, such that a sufficient number of transition amplitudes can interfere. At the quantum-classical interface, this corresponds to sufficiently small values of the effective Planck constant $\hbar_{\text {eff }}$, which is determined (via the uncertainty principle) by the comparison of $\hbar$ to the typical scales of the given problem, measured in canonical action-angle variables [7].

Besides simple billiard shaped cavities, which are of some relevance in the context of microdisc lasers [8], strongly perturbed atomic [0, 9] and molecular [10] systems are perfect candidates to study signatures of Anderson localization in quantum systems without disorder. The simplest (though realistic) representative of the latter is the hydrogen atom exposed to electromagnetic fields [7], with the atom initially prepared in a Rydberg level of principal quantum number $n_{0} \gg 1$, and a driving field frequency $\omega \sim n_{0}^{-3}$ near resonant with the atomic transition $n_{0} \rightarrow n_{0}+1$, i.e., within the microwave range. Such choice of the relevant parameters satisfies both general requirements stated above: on the one hand, the ionization potential of a Rydberg state $\left|n_{0}\right\rangle$ (we neglect the angular degree of freedom in our present treatment) requires the (net) absorption of approximately $N \simeq 1 / 2 n_{0}^{2} \omega \sim n_{0}$ photons to establish a transition to the atomic continuum. If, much as in the solid-state problem, each atomic bound state $n>n_{0}$ (with energy $E=-1 / 2 n^{2}$ ) which is quasi resonantly coupled to the initial state (i.e., $1 / 2 n_{0}^{2}-1 / 2 n^{2}=m \omega+\delta, m$ integer, and $\delta \ll \omega$ the detuning from resonance) plays the role of a single scatterer of the lattice, then a large number of transition amplitudes between $\left|n_{0}\right\rangle$ and the continuum become available (notice that emission events will eventually couple states with $n<n_{0}$ as well). This number rapidly increases with $N$, which therefore plays the role of the "atomic sample size", in analogy to the length $L$ of a solid-state sample [11]. On the other hand, due to the nonlinearity of the Coulomb potential, the detuning $\delta$, which determines the coupling strength between quasi resonantly coupled states, will be effectively randomized (much alike the simple generation of random numbers by a mod operation [12]), and this accounts 
for randomizing the phases of the various transition amplitudes which mediate the ionization process [13]. Consequently, the general scenery for Anderson localization to occur - this time on the energy axis rather than along the lattice - is set. In addition, a perfect classical analogue exists for the driven hydrogen atom: It is well-established that ionization is brought about by classical chaos, in the specified parameter range, since the quasi resonant coupling of sequences of Rydberg states described above destroys the good quantum numbers of the problem, which is synonymous to nonintegrability on the classical level [7, 9].

Indeed, the above analogy between charge transport through disordered solids and the ionization of Rydberg states by microwave fields has been identified approximately 20 years ago [ [ , 5, 6, 7]. Baptized "dynamical localization" (to stress its origin in dynamical chaos rather than in disorder) it has been qualitatively demonstrated by several independent experimental groups, on various atomic species [14, 15, 16, 17, 18]. A theoretical framework - known as "photonic localization theory" [0] - which relies on an ingenious mixture of crude approximations on the atomic side and deep physical intuition on the statistical side, provides explicit expressions for the mean of the localization length

$$
\langle\xi\rangle=3.33 F_{0}^{2} \omega_{0}^{-10 / 3} n_{0}^{2},
$$

and for the sample size

$$
N=\frac{n_{0}}{2 \omega_{0}}\left(1-\frac{n_{0}^{2}}{n_{c}^{2}}\right),
$$

where the factor in parenthesis in (2) accounts for a shift of the ionization threshold to the finite value $n_{c}<\infty$, induced by experimentally unavoidable stray electric fields [17]. $\omega_{0}=\omega n_{0}^{3}$ and $F_{0}=F n_{0}^{4}$ are the frequency and amplitude scaled with respect to the classical Kepler frequency, and to the Coulomb field amplitude along the unperturbed classical Rydberg orbit, respectively [7].

According to the scaling theory of localization [19], $\xi$ fluctuates around $\langle\xi\rangle$ for different realizations of the sample, at finite $N$, and tends to the non-fluctuating, sample independent value $\langle\xi\rangle$ only for $N \rightarrow \infty$, with the statistical distribution of $\xi$ completely determined by the localization parameter

$$
\ell \equiv \frac{\langle\xi\rangle}{N} \simeq \frac{6.66 F_{0}^{2} n_{0}}{\omega_{0}^{7 / 3}}\left(1-\frac{n_{0}^{2}}{n_{c}^{2}}\right)^{-1} .
$$

By virtue of this last expression, different realizations of the same value of $\ell$ can be realized, at fixed $n_{0}$, by simultaneously tuning $F_{0}$ and $\omega_{0}$ over a finite interval. Since, for an exponentially localized wave function on the energy axis, the population close to threshold is $\sim \exp (-2 N / \xi)$, the "atomic conductance" $g$, and this is nothing but the total transition probability to the atomic continuum, should then reflect the fluctuations of $\xi^{-1}$ in exponentially enhanced fluctuations via

$$
g \sim \exp (-2 N / \xi)
$$


Consequently, Anderson localization of the electronic bound-space population of a periodically driven Rydberg state implies large scale fluctuations of $g$ and also of the total ionization yield, under changes of $\omega_{0}$, at fixed values of $\ell$ and $n_{0}$.

This latter prediction has been verified in a recent publication [11] on the conductance of periodically driven one-dimensional Rydberg states of atomic hydrogen. More precisely, [11] demonstrated the lognormal distribution of $g$ (which follows from a normal distribution of $\xi^{-1}$, via (4)), and the approximately linear dependence of $\operatorname{Var}(\ln g)$ on $\langle\ln g\rangle$ [20], for the single value $n_{0}=70$ of the principal quantum number. However, the following highly relevant questions remained unaddressed:

- Sample size $N$ and localization parameter $\ell$ explicitely depend on $n_{0}$, and therefore on $\hbar_{\mathrm{eff}} \sim n_{0}^{-1}$ (the latter relation is a direct consequence of the scale invariance of the classical equations of motion of the driven Rydberg electron [7]). In the light of our qualitative discussion above, $N$ and therefore $n_{0}$ must not be too small for the Anderson picture to prevail in the atomic ionization process, since otherwise not enough transition amplitudes with quasi random phases will contribute to the total ionization yield. Hence, are we able to detect significant deviations from the predictions of Anderson's model in the atomic problem, for smaller values of $n_{0}$ ?

- Which are the smallest values of $n_{0}$ for which the dominant signatures of Anderson localization remain detectable in the ionization process?

- Can we confirm the linear dependence of $\operatorname{Var}(\ln g)$ on $\langle\ln g\rangle$ for variable values of $n_{0}$ ?

- Under which conditions are the predicted fluctuations of the atomic conductance experimentally observable?

The present contribution attempts to answer these questions.

The paper is organized as follows: Section 2 summarizes our theoretical/numerical approach to the atomic problem at hand, and introduces our definition of the atomic conductance. In section 3 we investigate the statistical properties of the atomic conductance, and particularly their dependence on the principal quantum number $n_{0}$, which explicitely enters equations (1-3). Section 1 concludes the paper, with a discussion of the experimental implications of our results.

\section{Theoretical background}

The Hamiltonian of a hydrogen atom exposed to an electromagnetic field polarized along the $z$-axis reads, in atomic units:

$$
\mathrm{H}=\frac{1}{2} \vec{p}^{2}-\frac{1}{r}-\frac{F p_{z}}{\omega} \sin (\omega t) .
$$

Here, the dipole approximation in the velocity gauge was used, we dropped the ponderomotive energy shift, assumed an infinite mass of the nucleus, and neglected relativistic effects [21]. In the following, we shall furthermore restrict configuration 
space to the single dimension defined by the field polarization axis, which results in the Hamiltonian

$\mathrm{H}=\frac{1}{2} p_{z}{ }^{2}+V(z)-\frac{F p_{z}}{\omega} \sin (\omega t), \quad$ with $\quad V(z)=\left\{\begin{aligned}-\frac{1}{z}, & z>0 \\ \infty & , \quad z \leq 0\end{aligned}\right.$

This approximation was chosen to keep the numerical effort necessary for sampling sufficient statistical data within reasonable bounds, and is also justified for atoms initially prepared in extremal parabolic states which are quasi one-dimensional eigenstates of the unperturbed hydrogen atom [22, 23. Even for real three-dimensional atomic initial states with low angular momentum quantum numbers has this onedimensional model been shown to yield quantitatively satisfactory results, within a certain parameter regime [21,23]. Nonewithstanding, an extension of our present work to the real three-dimensional world remains clearly desirable and will bear further surprises, but is at present an extremely expensive enterprise which saturates the largest computer facilities currently available. For the time being, despite their restricted range of predictive power, our subsequent results exhibit enough novel phenomena which shed new light on the atomic ionization process, and indicate the road to follow in future 3D calculations.

In order to extract ionization yields from the time-periodic Hamiltonian (6), we exploit the Floquet theorem and diagonalize the Floquet Hamiltonian $\mathcal{H}=\mathrm{H}-\mathrm{i} \partial_{t}$ in a Sturmian basis, after complex dilation [21. This provides direct access to the poles of the resolvent of $\mathcal{H}$, and hence an exact representation of the Green's function and of the associated time evolution operator of our problem. The latter finally leads (after an average over the initial phase of the driving field) to the following expression for the atomic ionization yield $P_{\text {ion }}(t)$ as a function of the interaction time $t$ [21]:

$$
P_{\text {ion }}(t)=1-\sum_{\epsilon} w_{\epsilon} \exp \left(-\Gamma_{\epsilon} t\right), t>0
$$

The sum runs over a single Floquet zone of length $\omega$ on the energy axis [21], the $\Gamma_{\epsilon}$ represent the ionization rates of individual Floquet eigenstates $|\epsilon\rangle$ of the atom in the field, and the $w_{\epsilon}$ are their weights in the decomposition of the atomic initial state $\left|n_{0}\right\rangle$ over the Floquet basis. Note that for $n_{0} \simeq 40 \ldots 100$ approximately $50 \ldots 120$ Floquet states contribute with non-vanishing $w_{\epsilon}$ to the sum in (7). Therefore, we are in a situation which is profoundly different from the single-state approximation familiar from the ionization of atoms (initially prepared in their ground state) by intense optical fields [24.

From (77), we can now derive a definition of the atomic conductance $g$, in terms of the spectral information obtained from the diagonalization of $\mathcal{H}$, as the average ionization rate at $t \simeq 0[1]$ :

$$
\left.g \equiv \frac{1}{\Delta} \frac{\mathrm{d}}{\mathrm{d} t} P_{\text {ion }}(t)\right|_{t \simeq 0}=\frac{1}{\Delta} \sum_{\epsilon} \Gamma_{\epsilon} w_{\epsilon} .
$$

In order to render $g$ dimensionless, we divided by the average level spacing $\Delta$ of the Floquet eigenvalues. Furthermore, since in the atomic problem there is no incoming 
particle flux as in the solid-state transmission problem, it is reasonable to take the derivative at $t \simeq 0$, in the above expression. Note that the right-hand-side of (8) is strongly reminiscent of Landauer's formula for the conductance across a disordered sample [25], if we identify the Floquet rates $\Gamma_{\epsilon}$ with matrix elements of the transition matrix in the solid-state problem. Indeed, such an identification can be justified more formally, as we shall show elsewhere [26].

\section{Numerical results}

We have now set the scene for our statistical analysis of the atomic conductance. To gain a qualitative impression of the phenomenon we are dealing with, let us first focus on the parameter dependence of the ionization yield, equation (7), of the initial state $n_{0}=100$, for two different values $\ell=0.2$ and 1 , and an interaction time $t=300 \times 2 \pi / \omega[22$. Figure 1 shows our numerical result, within the interval $\omega_{0} \in[2.0 ; 2.5]$ (500 equidistant values of $\omega_{0}$ were found sufficient to resolve all structures of the signal). For $\ell=0.2$, the ionization yield is typically very small, close to zero, but is locally strongly enhanced (by orders of magnitude), at apparently random values of $\omega_{0}$. Also for $\ell=1$ the yield exhibits large fluctuations, however around a clearly finite average value larger than zero, and of the same order of magnitude as the average ionization probability. Both cases are reminiscent of conductance fluctuations through disordered solid-state samples, in the localized $(\ell=0.2)$ and in the diffusive (or delocalized, $\ell=1$ ) regime, respectively [27,28]. Note that the observed fluctuations occur on a scale $\delta \omega_{0} / \omega_{0} \simeq 10^{-2}$, i.e. $P_{\text {ion }}$ is a smooth function of $\omega_{0}$ on scales $\delta \omega_{0} / \omega_{0} \simeq 10^{-3}$ or smaller [29]. For $n_{0} \simeq 60 \ldots 100$, this corresponds to a typical frequency window of approximately $\delta \omega / 2 \pi \simeq 700 \ldots 150 \mathrm{MHz}$, on which the fluctuations should be detectable, rather than on scales smaller by one order of magnitude, as considered in [30].

To deduce the $\omega_{0}$-dependence of the atomic conductance from the yield displayed in figure 1, we still need to extract the average level spacing $\Delta$ from the raw numerical data. Since not all eigenstates $|\epsilon\rangle$ of $\mathcal{H}$ actually contribute to the ionization of $\left|n_{0}\right\rangle$, we have to account for the relative weight $w_{\epsilon}$ of the individual Floquet eigenstates in our definition of $\Delta$. One way of doing so is to estimate the number of effectively contributing Floquet states as $\exp \left(W_{\text {Shannon }}\right)$, via the Shannon entropy $W_{\text {Shannon }}=-\sum_{\epsilon} w_{\epsilon} \ln w_{\epsilon}[31$. Another way [11] is to assume that as many Floquet states contribute to the ionization dynamics as there are quasi resonantly coupled unperturbed states between the atomic initial state and the continuum threshold, i.e. $N$ (see equation (2) above). Both estimates consistently provide similar results, i.e.,

$$
\Delta \simeq \frac{\omega}{N} \simeq \frac{\omega}{\exp \left(W_{\text {Shannon }}\right)}
$$

as illustrated in figure 2, in the localized as well as in the delocalized regime (note that, more precisely, $N>\exp \left(W_{\text {Shannon }}\right)$ in the localized regime, and $N<\exp \left(W_{\text {Shannon }}\right)$ in the delocalized regime, what is consistent with the interpretation of $N$ as the atomic sample size). We checked that this remains true for all values of $n_{0}$ considered hereafter, 
and that the statistical properties of $g$ are insensitive to the definition of $\Delta$ we choose, except for an irrelevant offset. Therefore, all subsequent results are presented with the convention $\Delta \equiv \omega / N$.

With this definition, we show the $\omega_{0}$-dependence of the atomic conductance in figure 3, for two values of $n_{0}=40,100$, as well as for the two values of the localization parameter already employed in figure 11. Clearly, the erratic fluctuations of $P_{\text {ion }}$ carry over to the atomic conductance. Note that in the localized regime $(\ell=0.2)$ the fluctuations manifest on a logarithmic scale $\left(\ln g\right.$ is plotted vs. $\omega_{0}$ on the left column of figure 3), whereas the fluctuations of $g$ are of the same order of magnitude as its average value, in the delocalized regime $(\ell=1$, right column of figure $3, g$ is plotted vs. $\left.\omega_{0}\right)$. Furthermore, the scale $\delta \omega_{0} / \omega_{0}$ of the fluctuations clearly becomes finer as the principal quantum number $n_{0}$ is increased from $n_{0}=40$ to $n_{0}=100$, an observation which is consistent with the increased density of states as one approaches the ionization threshold.

It should be stressed here that such large fluctuations, especially those in the localized regime, are truely remarkable, since they manifest in a quantity which represents a weighted average over the entire Floquet spectrum, according to equation (8). In an experiment with, say, $n_{0}=80$, and a carrier frequency of approximately $\omega / 2 \pi \simeq 30 \mathrm{GHz}$, a detuning of approximately $100 \mathrm{MHz}$ can enhance the ionization yield from virtually zero to more than 10\%! It has been shown earlier [29, 32] that specific, individual Floquet eigenstates of the atom in the field may exhibit large scale, erratic fluctuations of their ionization rates under changes of some control parameter, for instance of $\omega_{0}$. Here, it is the conspiracy of the distribution of the weights $w_{\epsilon}$ and rates $\Gamma_{\epsilon}$ over the entire spectrum which produces a similar effect!

Let us now proceed to a first quantitative test of photonic localization theory. If the atomic localization parameter $\ell$ defined in (3) indeed plays the same crucial role as in the solid-state problem, then $\langle\ln g\rangle$ should decrease linearly with increasing $\ell^{-1}$, by virtue of (3, 4 ). Figure 4 shows our numerical result, for different values of $n_{0}$. Apparently, the solid-state prediction is almost perfectly followed for the largest principal quantum number (i.e., the largest individual values of $\langle\xi\rangle$ and $N$ in (1, 2) ). On the other hand, the smaller $n_{0}$, and, hence, the smaller $\langle\xi\rangle$ and $N$, the larger are the deviations from the linear dependence. This, however, can be readily understood since the deviations systematically (for all values of $n_{0}$ ) occur for localization parameters (small $\ell$, large $\ell^{-1}$ ) which correspond to average localization lengths $\langle\xi\rangle<3$ (down to $\langle\xi\rangle \simeq 0.6 \ldots 0.75$, for $\ell=0.1$ and $\left.n_{0}=40\right)$. Then, according to the simple picture developed in the introduction, no more than two bound states of the atom are efficiently coupled by the driving field, and it does not make any sense to speak of an electronic wave function which is exponentially localized over quasi resonantly coupled bound states on the energy axis. As a matter of fact, it is rather surprising that the linear behaviour is observed in figure 1 for values of $\langle\xi\rangle$ as small as 3 or 4 , since the assumptions [7] for the derivation of (1) imply $\langle\xi\rangle \gg 1$.

A further quantitative test of the analogy between atomic and solid-state transport 
problem is the statistical distribution of the atomic conductance sampled over different values of $\omega_{0}$, for fixed $\ell$. Figures 5 and 6 show histograms of $\ln g$, for $n_{0}=40,100$, respectively, and $\ell=0.1 \ldots 0.5$ (for each histogram, approx. $10000 \ldots 60000$ resonances with nonvanishing weights $w_{\epsilon}$ contribute to 500 values of $g$ ). Systematically, the lognormal fits of the histograms obtained from our data improve as $n_{0}$ is increased, in particular in the wings of the distributions. For small $\ell$, low values of $n_{0}$ tend to induce a sharp cut-off at small conductances, what we attribute once more to the increasing "granularity" of the ionization process as $n_{0}$ decreases. For principal quantum numbers $n_{0} \geq 70$, however, the distributions of the atomic conductance are well fitted [33] by the lognormal prediction derived from the Anderson model.

For given $n_{0}$ and growing $\ell$, the distribution of $\ln g$ shifts to larger values, as visible, e.g., in figures 6 and 0 , for $n_{0}=100$, and also from figure 4 . Furthermore, as already could be expected from the comparison of the $\omega_{0}$-dependence of the atomic conductance for $\ell=0.2$ and $\ell=1$ (figure 3), the lognormal fit ceases to be a good approximation of the histograms for too large values of $\ell$. As evident from figure 7, the distribution starts to get asymmetric at $\ell=1$, and is clearly not lognormal any more for $\ell=2$. This transition from the localized to the delocalized (or diffusive [0, [0]) regime is even more pronounced in figure 8, where we plot the histogram of $g$ rather than of $\ln g$ : with $\ell$ increasing from 0.75 to 2 , the distribution shifts to larger values, broadens, and develops a large gap at $g=0$. Still, a Gaussian distribution of $g$ as observed for diffusive transport in the solid-state transmission problem [20] cannot be established here.

Finally, we examined the variances of $\ln g$ as obtained from our numerical data, for different values of $n_{0}$. The result is shown in figure 9. Whereas localization theory suggests a linear dependence $\operatorname{Var}(\ln g) \sim-\langle\ln g\rangle$ [20], our data appear to support this expectation only within a finite interval of $\langle\ln g\rangle$, which furthermore depends on $n_{0}$, and can be roughly confined by the limits $-12 \leq\langle\ln g\rangle \leq-7$. For small values of $\langle\ln g\rangle$, the variance systematically drops faster than linearly, and it turns out that its overall dependence on the average conductance is best fitted by a quadratic law, for all $n_{0}$. Once again, we attribute this deviation from the solid-state picture to the finite size effect which already manifested itself in the dependence of $\langle\ln g\rangle$ on $\ell^{-1}$, and in the distribution of $\ln g$, for small values of $\ell$. For too small localization lengths, the distribution of $\ln g$ is not lognormal any more. Deviations notably occur in the wings, and we cannot expect a linear variation of $\operatorname{Var}(\ln g)$ in this parameter regime. On the other extreme, at large localization parameters, the variance saturates, in accordance with our observations in figures 3, 4, and 8, as well as with general expectations for diffusive transport in disordered solids [3].

\section{Conclusions}

To summarize, we can now respond to the questions we formulated at the beginning of this paper. Our above results demonstrate that the essential statistical features of the conductance across an Anderson-localized solid-state sample indeed carry over to the 
fully deterministic ionization process of quasi one-dimensional hydrogen Rydberg states under microwave driving, where dynamical chaos substitutes disorder. In response to our first introductory question, we do observe important amendments to the solid-state picture (which implicitly always assumes a localization length much larger than the typical distance between neighbouring lattice sites) imported to the atomic realm by photonic localization theory. These amendments directly originate - via the atomic sample size $N$, equation (2) - in the finite size of $\hbar_{\text {eff }} \sim n_{0}^{-1}$. However, they do neither affect the monotonous decrease of the mean atomic conductance with the inverse of the localization parameter (figure 4 ), nor the large-scale fluctuations of $g$, for different realizations of $\ell$.

A rough estimate allows an answer to our second question: Figures 79 suggest that the transition from localized to diffusive transport sets in at $\ell \geq 0.5$. Furthermore, we have seen that deviations from the exponential dependence of $\langle\ln g\rangle$ on $\ell^{-1}$ get manifest for $\langle\xi\rangle<3$. Considering the monotonous decrease of $\langle\ln g\rangle$ with decreasing $\ell$ (figure 1 ) as the most robust signature of localization, we therefore require that the minimum value of $n_{0}$ permits localization lengths $\langle\xi\rangle>2$ at $\ell=0.5$. By virtue of (1-13), this implies $n_{0} \simeq 4 \omega_{0}\langle\xi\rangle>8 \omega_{0} \simeq 16 \ldots 20$ (with $\omega_{0}=2.0 \ldots 2.5$ ), and is consistent with earlier numerical results [21] for smaller values of $n_{0} \simeq 23$. On the other hand, however, the exponentially enhanced fluctuations of the atomic conductance, as the actual, quantitative fingerprint of Anderson localization in our driven atomic system, do fully prevail only for $n_{0}>70$ (figures 5-7, see also [33]).

The reply to our third question directly follows from our discussion of figure 9 above, which suggests a smooth transition from lognormally distributed atomic conductances for sufficiently large localization lengths and principal quantum numbers, to more coarse-grained distributions with larger variances. Taking into account the perturbative coupling limit defined by small values of $\langle\xi\rangle \simeq 2$, it should be possible to derive an approximate analytical expression for the general behaviour of $\operatorname{Var}(\ln g)$ with $\langle\ln g\rangle$.

Finally, we verified that the fluctuations displayed in figure 3 remain essentially unaffected if we average over a finite window of $F_{0}$ (and, hence, of $\ell$ ), at any given value of $\omega_{0}$. We assumed a relative error of $\delta F_{0} / F_{0} \leq 5 \%$ in the experimental calibration of the field amplitude experienced by the atoms, which is state of the art in laboratory experiments [17, 18]. Therefore, exponentially large fluctuations of the atomic conductance in the localized regime should be observable via measurements of the ionization yield at sufficiently short interaction times (see figures 1 and 3). Importantly, such experiments must be performed below the ionization (i.e., delocalization) threshold, as immediately apparent from figure 1. Since, so far, most experimental evidence in support of dynamical localization in periodically driven atoms is based on measurements of the ionization threshold [14, 15, 17, 18], which doesn't prove more than a monotonous decrease of the average conductance with decreasing $\ell$, the crucial experimental test of Anderson localization in driven atomic systems is yet to be performed. 


\section{Acknowledgments}

We thank Uzy Smilansky for his warm hospitality during a visit at the Weizmann

Institute of Science, where part of this work was accomplished. Financial support by the Minerva Foundation (AB), and by the Studienstiftung des dt. Volkes (SW) is gratefully acknowledged. We also are grateful to Jakub Zakrzewski, for valuable discussions and for making available his Gaussian fit routine.

\section{References}

[1] Mesoscopic quantum physics, edited by Akkermans E, Montambaux G, Pichard J L, and ZinnJustin J (North-Holland, Amsterdam, 1995).

[2] Anderson P W, Phys. Rev. 109, 1492 (1958).

[3] Kramers B and MacKinnon A, Rep. Prog. Phys. 56, 1469 (1993).

[4] Casati G, Chirikov B V, Ford J, and Izrailev F M, in Stochastic Behavior in Classical and Quantum Hamiltonian Systems, edited by G. Casati and J. Ford (Springer-Verlag, Berlin, 1979), p. 334.

[5] Fishman S., Grempel D R, and Prange R E, Phys. Rev. Lett. 49, 509 (1982).

[6] Casati G, Chirikov V, and Shepelyansky D L, Phys. Rev. Lett. 53, 2525 (1984).

[7] Casati G, Chirikov V, Shepelyansky D L, and Guarneri I, Phys. Rep. 154, 77 (1987).

[8] Starykh Q A, Jacquod P R, Narimanov E E, and Stone A D, Phys. Rev. E 62, 2078 (2000).

[9] Delande D and Buchleitner A, Adv. At. Mol. Phys. 34, 85 (1994).

[10] Leitner D M and Wolynes P G, Chem. Phys. Lett. 276, 289 (1997).

[11] Buchleitner A, Guarneri I, and Zakrzewski J, Europhys. Lett. 44, 162 (1998).

[12] Press W H, Teulosky S A, Vetterling W T, and Flannery B P, Numerical Recipes in FORTRAN: the art of scientific computing, 2 ed. (Cambridge University Press, Cambridge, 1994).

[13] Casati G, Guarneri I, and Shepelyansky D L, Physica A 163, 205 (1990).

[14] Galvez E J, Sauer J E, Moorman L, Koch P M, and Richards D, Phys. Rev. Lett. 61, 2011 (1988).

[15] Bayfield J E, Casati G, Guarneri I, and Sokol D W, Phys. Rev. Lett. 63, 364 (1989).

[16] Blümel R, Buchleitner A, Graham R, Sirko L, Smilansky U, and Walther H, Phys. Rev. A 44, 4521 (1991).

[17] Arndt M, Buchleitner A, Mantegna R N, and Walther H, Phys. Rev. Lett. 67, 2435 (1991).

[18] Noel W M, Griffith W M, and Gallagher T F, Phys. Rev. A 62, 063401 (2000).

[19] Abrahams E, Anderson P W, Licciardello D C, and Ramakrishnan T V, Phys. Rev. Lett. 42, 673 (1979); Efetov K B, Adv. Phys. 32, 53 (1983); Stone A D, in [1], and references therein.

[20] Pichard J L, Zanon N, Imry Y, and Stone A D, J. Phys. (Paris) 51, 587 (1990).

[21] Buchleitner A, Delande D, and Gay J C, J. Opt. Soc. Am. B 12, 505 (1995).

[22] Koch P W and van Leeuwen K, Phys. Rep. 255, 289 (1995).

[23] Buchleitner A and Delande D, Phys. Rev. A 55, R1585 (1997).

[24] Gavrila M, in Atoms in Intense Laser Fields, Vol. 1 of Advances in atomic, molecular, and optical physics, supplements, edited by M. Gavrila (Academic Press, Boston, 1992), p. 435.

[25] Landauer R, Philos. Mag. 21, 863 (1970).

[26] Wimberger S and Buchleitner A (unpublished).

[27] Washburn S and Webb R A, Adv. Phys. 35, 375 (1986).

[28] Popovic D, Fowler A B, Washburn S, and Stiles P J, Phys. Rev. B 42, 1759 (1990).

[29] Buchleitner A and Delande D, Chaos, Solitons \& Fractals 5, 1125 (1995).

[30] Sirko L, Arndt M, Koch P M, and Walther H, Phys. Rev. A 49, 3831 (1994).

[31] Blümel R and Smilansky U, Phys. Rev. Lett. 58, 2531 (1987).

[32] Zakrzewski J, Delande D, and Buchleitner A, Phys. Rev. E 57, 1458 (1998).

[33] Wimberger S, Diploma thesis, Ludwig-Maximilians-Universität München, München, 2000. 

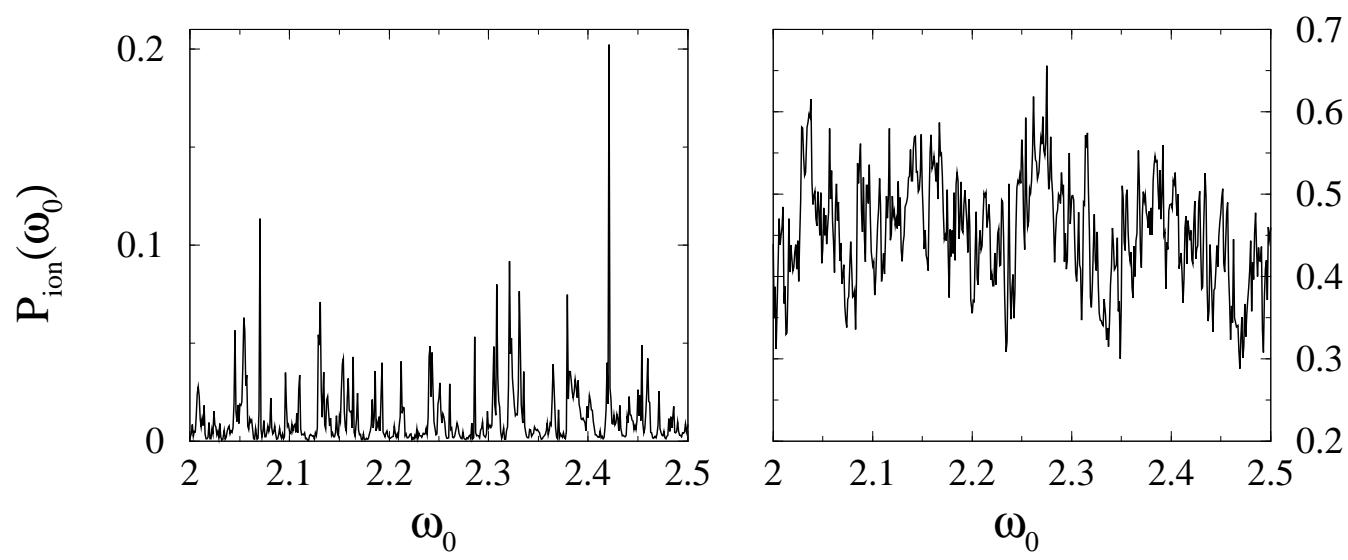

Figure 1: Ionization yield $P_{\text {ion }}(t)$ vs. the scaled frequency $\omega_{0}$, for an initial principal quantum number $n_{0}=100$, and fixed interaction time $t=300 \times 2 \pi / \omega$. In the localized regime $(\ell=0.2$, left), the ionization yield is close to zero, amended by erratic fluctuations. In the delocalized regime $(\ell=1$, right), the average yield is clearly finite, with erratic fluctuations of the same order of magnitude.

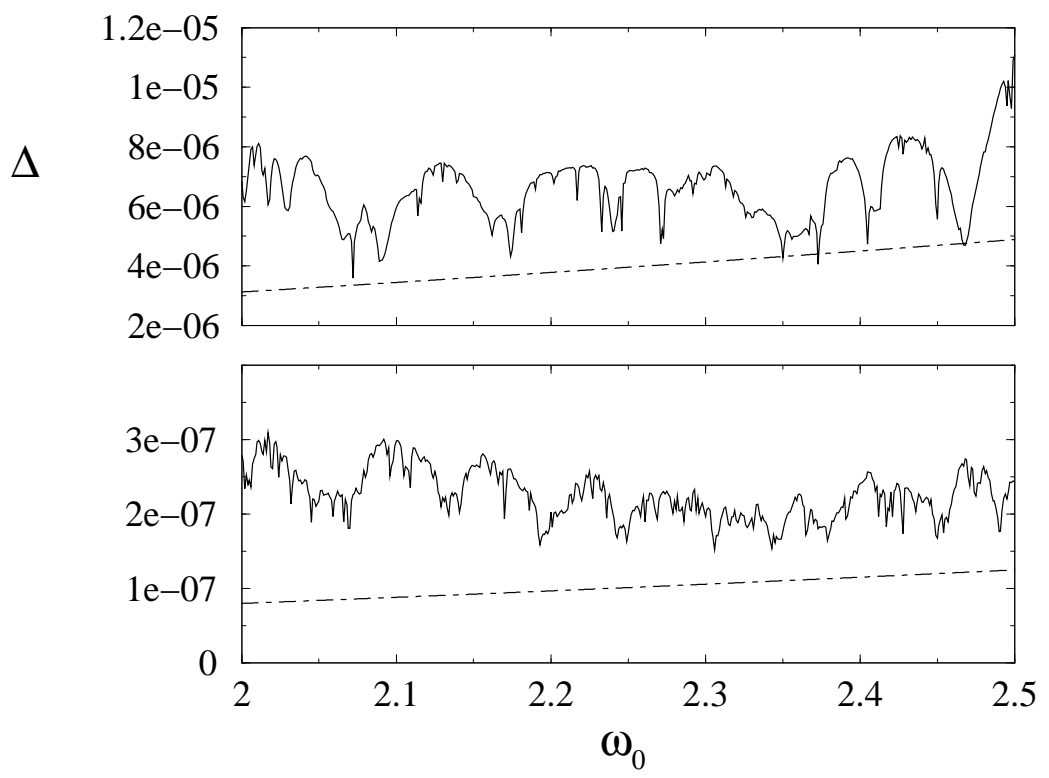

Figure 2: Comparison of the two estimates of the average level spacing $\Delta$ (equation (9)), as a function of the scaled frequency $\omega_{0}$, for constant localization parameter $\ell=0.2$. The full curves show the spacings deduced from the Shannon entropy $W_{\text {Shannon }}$, which in the localized regime $(\langle\xi\rangle<N)$ are typically larger than those given by the simple estimate $\omega / N$ (dashed-dotted lines). As the quantum number $n_{0}$ of the initial Rydberg state increases from 40 (top) to 100 (bottom), the absolute values of the spacings decrease. The statistical properties of the atomic conductance (8) discussed hereafter turn out to be independent of the definition of $\Delta$, except for an irrelevant offset in the statistical distributions. 

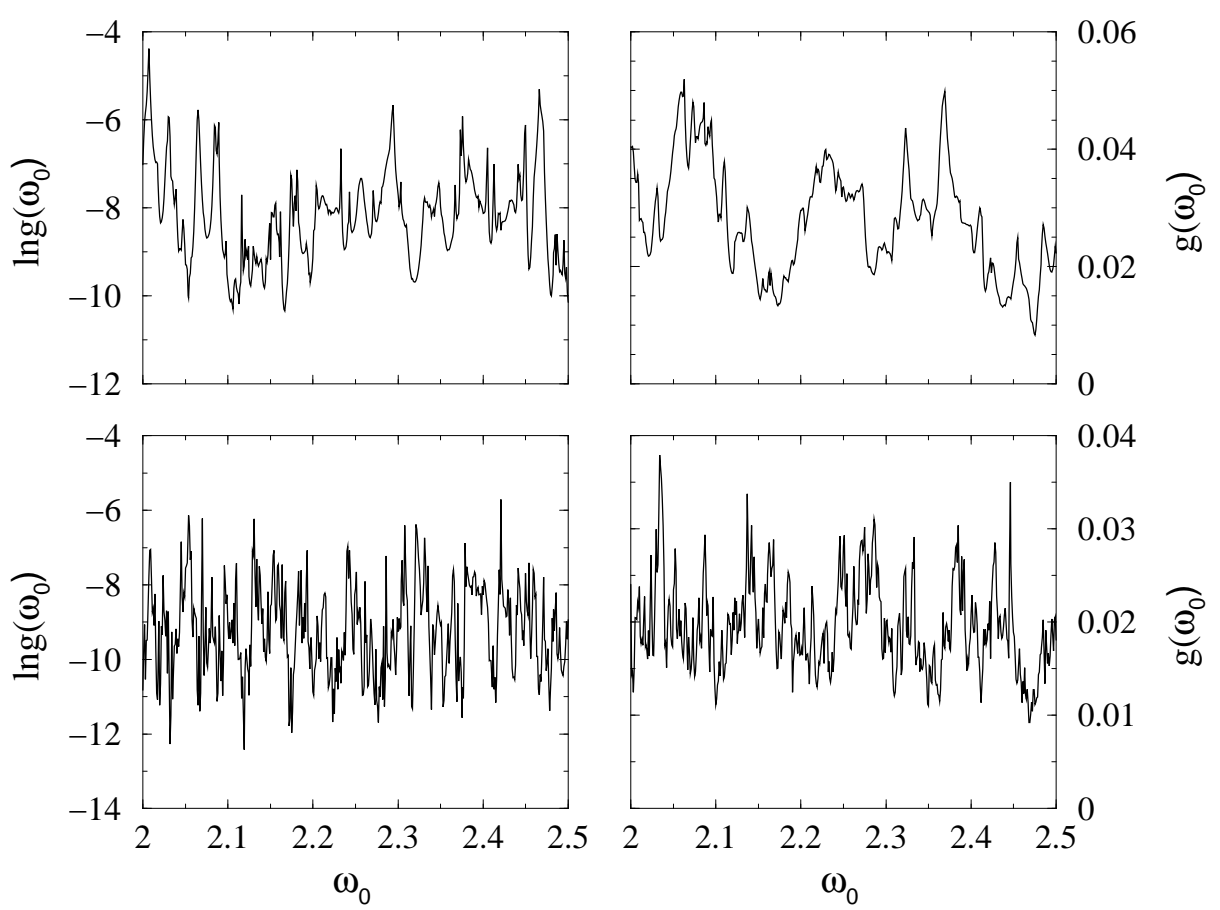

Figure 3: Atomic conductance vs. scaled frequency $\omega_{0}$, for localization parameters $\ell=0.2$ (left), 1 (right), and for initial atomic Rydberg states $n_{0}=40$ (top), $n_{0}=100$ (bottom), respectively. The semi-logarithmic plots for the localized case $\ell=0.2$ (left column) clearly exhibit huge fluctuations over several orders of magnitude, a characteristic feature of quantum transport in the presence of Anderson localization (see text). In the delocalized regime $(\ell=1$, right column) the amplitude of the fluctuations is strongly reduced (note the linear scale) and comparable to the average conductance. 


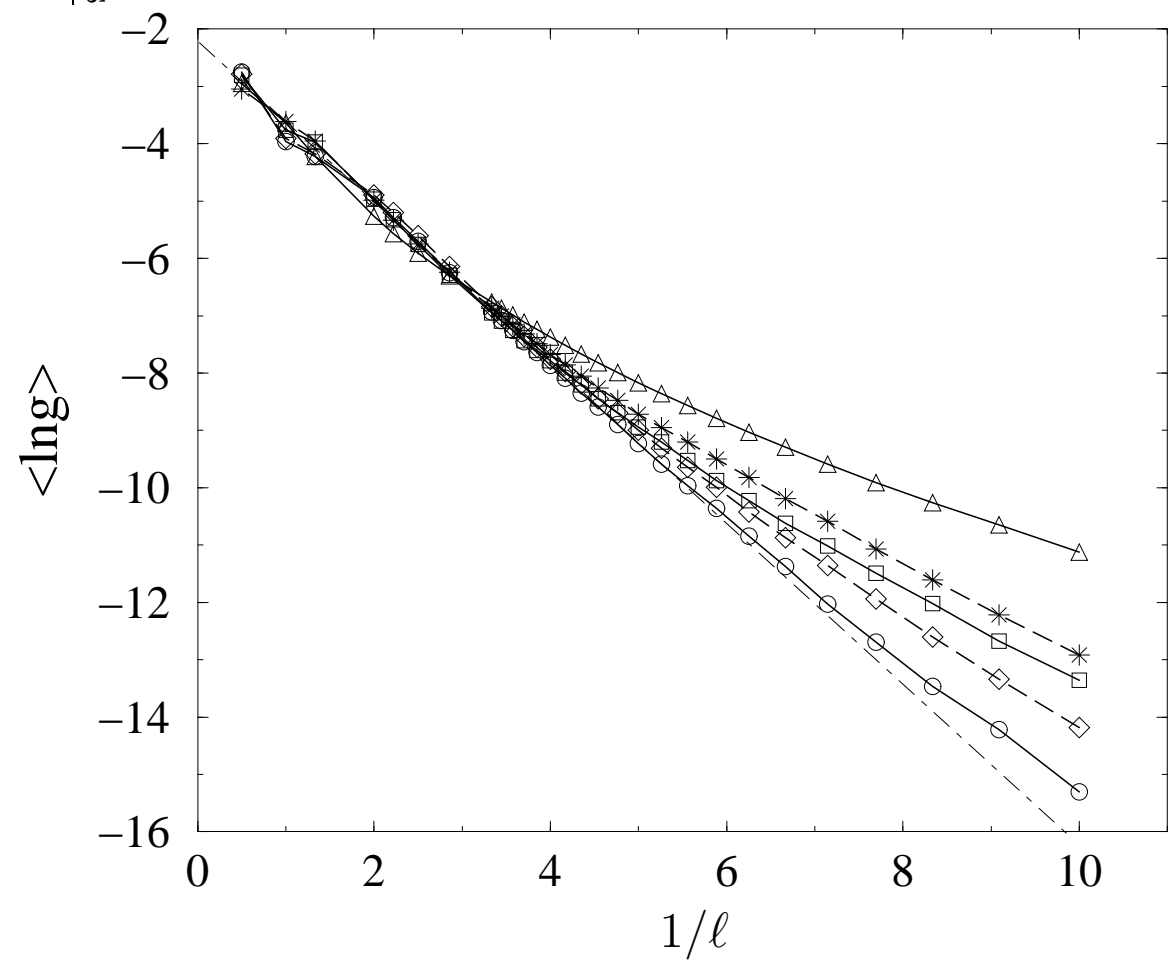

Figure 4: Average value of $\ln g$ vs. the inverse localization parameter $\ell^{-1}$, for principal quantum numbers $n_{0}=40(\triangle), 60(\star), 70(\square), 90(\diamond), 100(\circ)$ of the atomic initial state, and $\ell=0.1 \ldots 2$. $\langle\ln g\rangle$ was obtained from sampling $g$ for 500 equidistant values of $\omega_{0} \in[2.0 ; 2.5]$, at fixed $\ell$ (see equation (3)). For $n_{0}=100$ we observe an almost perfectly linear dependence, in agreement with (4) and, hence, with the Anderson picture. As $n_{0}$ decreases, $\langle\xi\rangle$ and $N$ decrease at fixed $\ell$, and a clear deviation from an exponential decrease of $\langle\ln g\rangle$ with $\ell^{-1}$ is systematically observed for $\langle\xi\rangle<3$. 

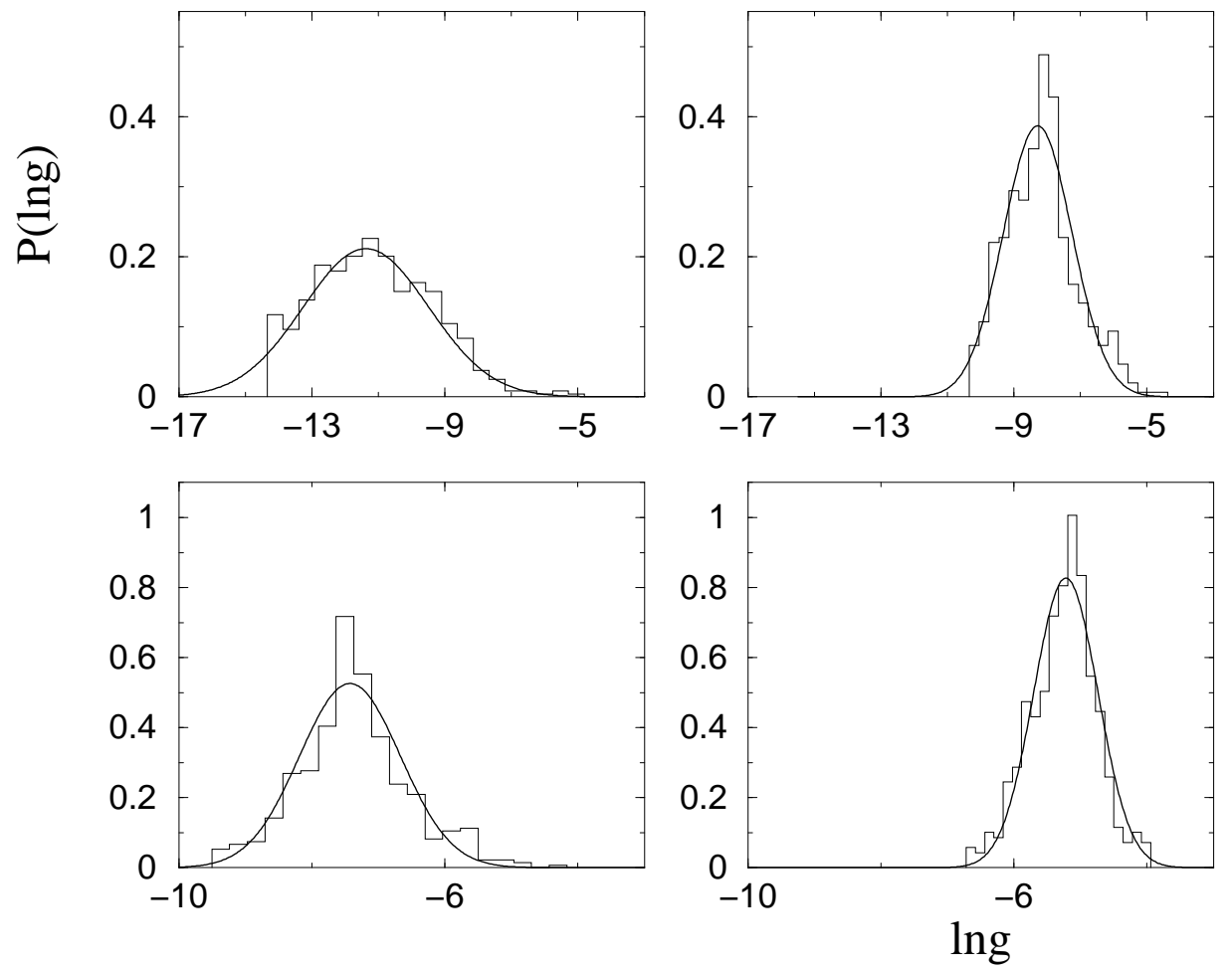

Figure 5: Distributions of the logarithm of the atomic conductance $\ln g$ sampled over 500 equidistant values of $\omega_{0} \in[2.0 ; 2.5]$, for each value of the localization parameter $\ell=0.1$, $0.2,0.25,0.5$, (top left to bottom right), and $n_{0}=40$. The thick lines show the best fit to a normal distribution which is expected on the grounds of Anderson localization theory. The histograms shift to higher values of $\ln g$, with decreasing widths as $\ell$ grows. Clearly, for this smallest $n_{0}$-value employed in our calculations, there are considerable deviations from the expected lognormal behaviour. 

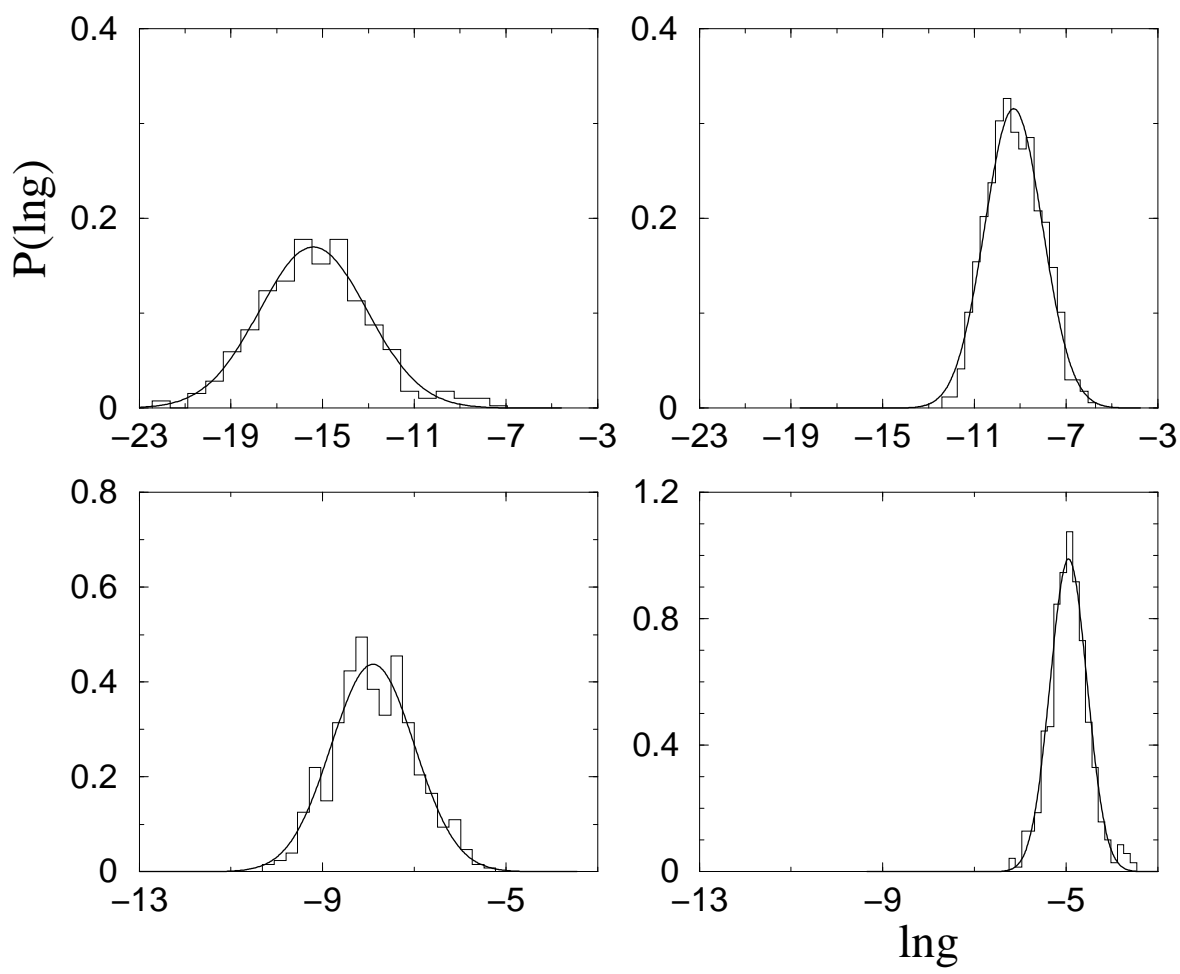

Figure 6: Histograms of $\ln g$ fitted by a normal distribution (thick line), for $n_{0}=100$ and the same localization parameters and sampling interval as in figure 5. The agreement with the lognormal prediction implied by Anderson localization theory is essentially perfect now, at sample sizes and average localization lengths $N \simeq 15 \ldots 19$ and $\langle\xi\rangle \simeq 2 \ldots 9$, respectively, for $\ell=0.1 \ldots 0.5$. 

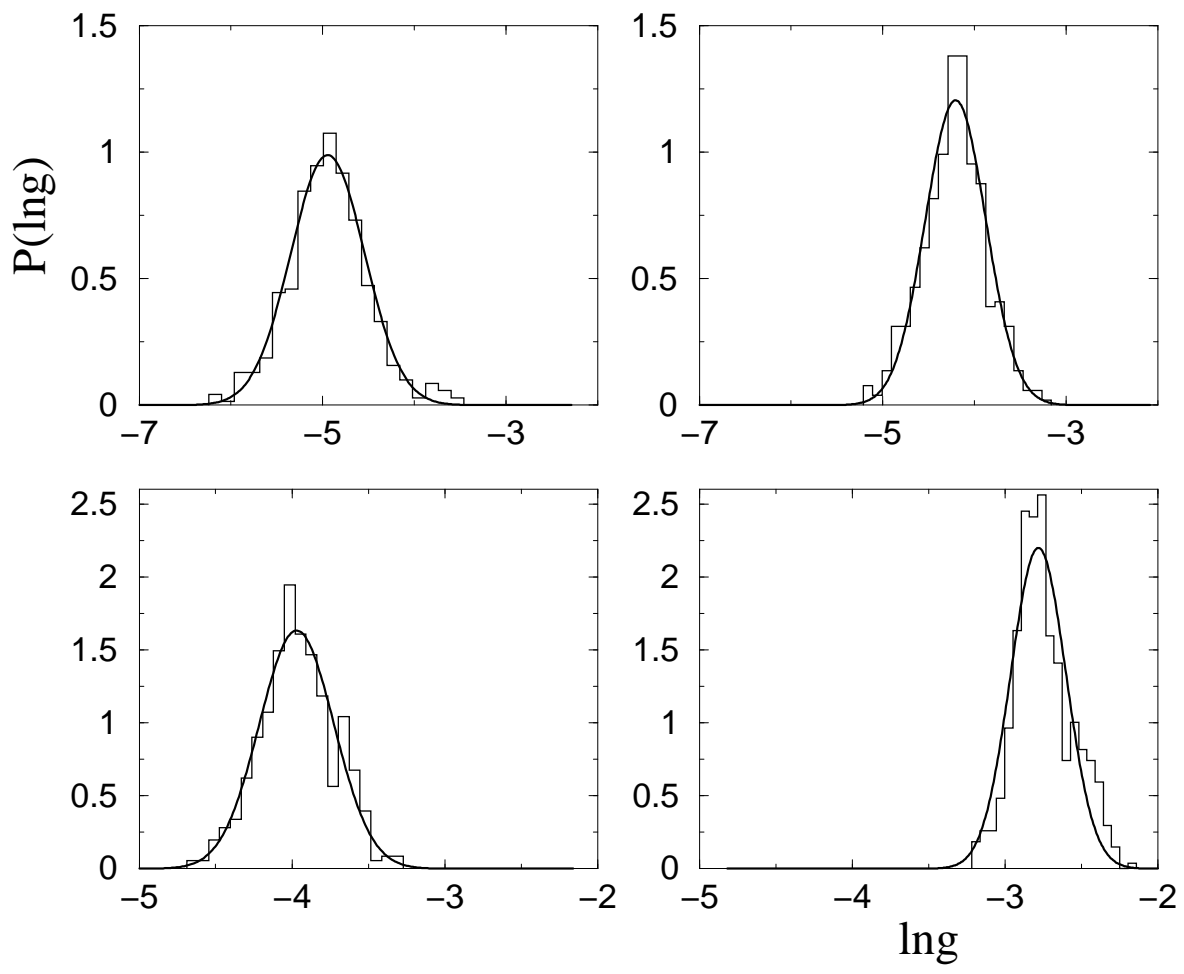

Figure 7: Distributions of the logarithm of the atomic conductance for $n_{0}=100$ and localization parameters $\ell=0.5 \ldots 2$ (top left to bottom right). The distributions shift to larger values of $\ln g$ and get narrower as $\ell$ is increased. At $\ell=2$, a clear deviation from the lognormal fit is observed, which reveals the transition to the delocalized regime. Similar results are obtained over the entire range $n_{0}=40 \ldots 100$.

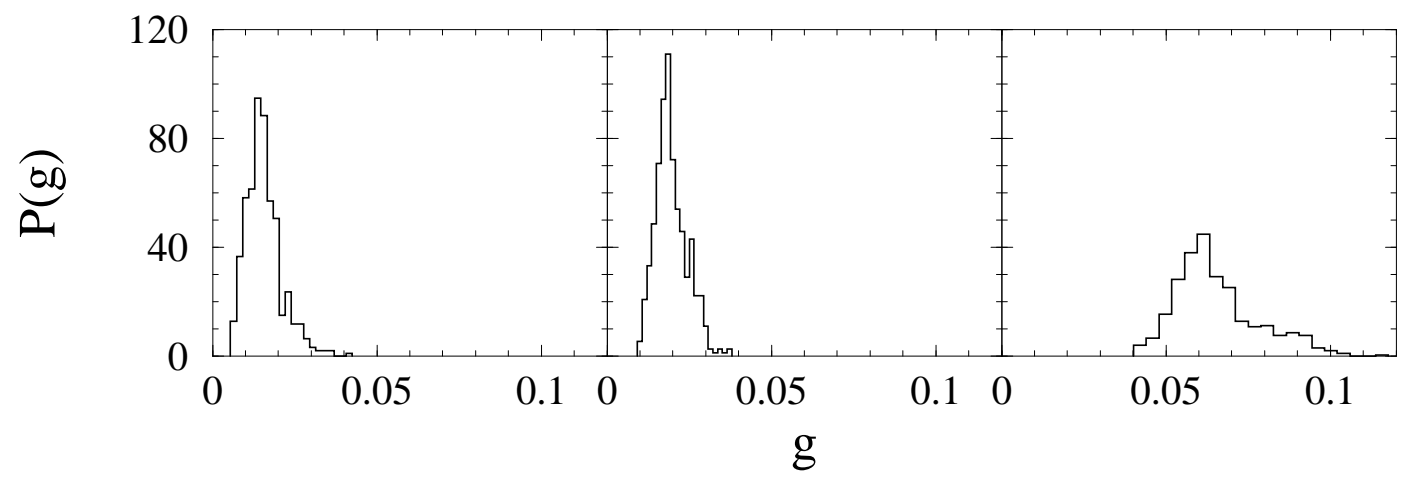

Figure 8: Distributions of the atomic conductance of $n_{0}=100$, for large localization parameters $\ell=0.75$ (left), 1 (middle), and 2 (right), as in figure 7 , but on a linear scale. The broadening of the distribution with increasing $\ell$, together with the widening gap at $g=0$, indicates the transition to the delocalized (diffusive) regime. 


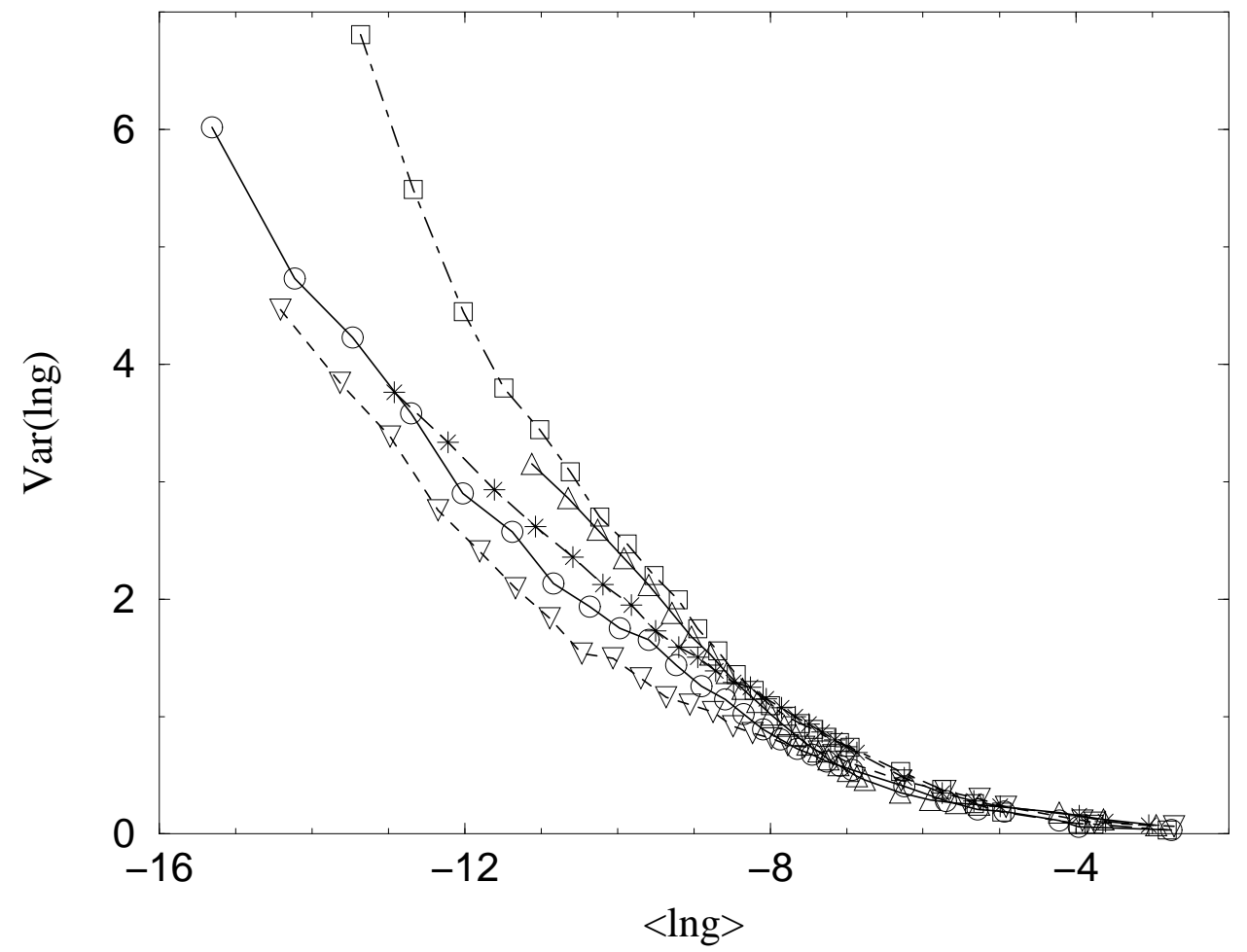

Figure 9: Variances of the atomic conductance $\ln g$ vs. its average $\langle\ln g\rangle$, for $n_{0}=40(\triangle)$, $60(\star), 70(\square), 80(\nabla)$, and $100(\circ)$, respectively. The localization parameter $\ell$ increases from left to right $(\ell=0.1 \ldots 2$. $)$. Each data point was obtained by sampling $g$ over 500 equidistant values of $\omega_{0} \in[2.0 ; 2.5]$, for given $\ell$. 